\title{
On the uniqueness of the solution of the cost minimization problem with generalized Sato production function
}

\author{
Vedran Kojić ${ }^{1, *}$, Zrinka Lukač ${ }^{1}$ and Krunoslav Puljić ${ }^{1}$ \\ ${ }^{1}$ Faculty of Economics and Business, University of Zagreb \\ Trg J. F. Kennedyja 6, 10000 Zagreb, Croatia \\ E-mail: 〈\{vkojic, zlukac, kpuljic\}@efzg.hr〉
}

\begin{abstract}
Whenever a firm is maximizing its profit, it necessarily has to minimize its cost. Thus, the cost minimization problem is one of the central problems in the theory of the firm. When presenting this problem, the majority of microeconomic textbooks use very well-known production functions, such as Leontief, Cobb-Douglas, or other CES production functions. The goal of this paper is to analyze the cost minimization problem with the generalized Sato production function. The generalized Sato production function is one of the non-standard production functions with variable elasticity of substitution. First, we show that the generalized Sato production function is continuous, strictly monotone, strictly quasiconcave and that a positive amount of output requires positive amounts of some of the inputs. Next, by using mathematical programming we show that the cost minimization problem with generalized Sato production function has a unique solution. This result is very important since it implies the existence of the corresponding cost function and conditional input demands.
\end{abstract}

Keywords: generalized Sato production function, cost minimization problem, unique solution

Received: December 22, 2020; accepted: February 07, 2021; available online: June 29, 2021

DOI: $10.17535 /$ crorr.2021.0004

\section{Introduction}

The most general way to characterize the firm's technology is the production possibility set. Often, however, it is considered that firms producing only a single product from many inputs. Thus, a production function is a more convenient way of describing the firm's technology (see [4]). Furthermore, as opposed to using the more general production possibility set, using a production function in the analysis is very special, but theoretically very important. To illustrate the stability conditions of the growth equilibrium, a new production function (1) was introduced at the meeting of the Econometric Society in 1963 by professor Ryuzo Sato (see [10]):

$$
Y=F(K, L)=\frac{K^{2} L^{2}}{a K^{3}+b L^{3}},
$$

where $a>0, b>0, Y$ is the total output, $K$ is the capital, and $L$ is the labor (see the Figure 1). One of the recent applications of the function (1) can be found, for example, in [8].

In microeconomic theory, the production function, $f$, is a mapping from $\mathbb{R}_{+}^{n}$ into $\mathbb{R}_{+}$. Furthermore, some properties of the production function are assumed as follows (see [4]).

${ }^{*}$ Corresponding author. 
Assumption 1. The production function, $f: \mathbb{R}_{+}^{n} \rightarrow \mathbb{R}_{+}$is continuous, strictly increasing, and strictly quasiconcave on $\mathbb{R}_{+}^{n}$, and $f(0)=0$.

The proof that the function (1) satisfies the properties in Assumption 1 is given in [5].
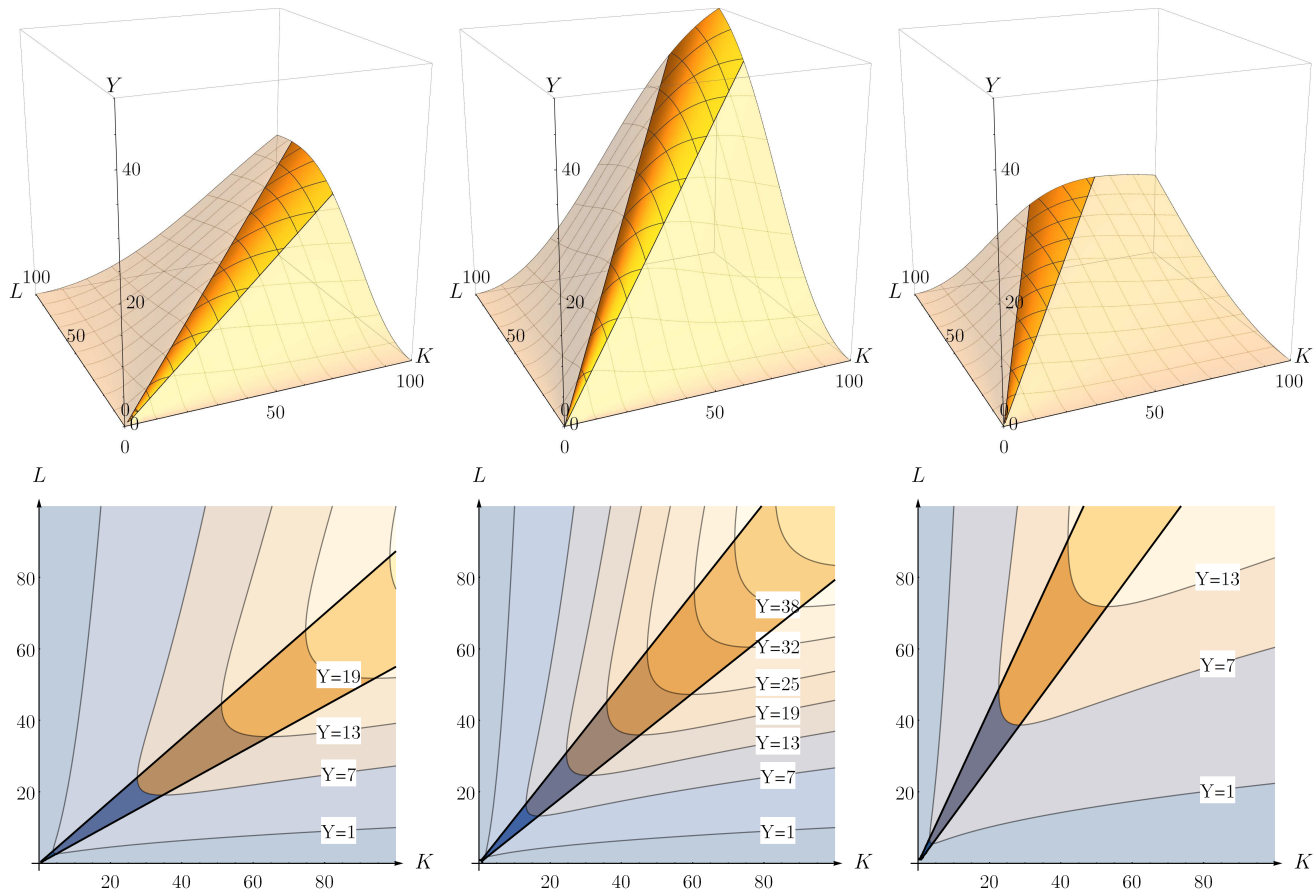

Figure 1: The $3 D$ and $2 D$ graphs of the Sato function from (1), with $(a, b) \in\{(1,3),(1,1),(5,1)\}$

In 1969, the generalization of the Sato production function (1), called the generalized Sato production function, given by equation (2) in Section 3 of this paper, was proposed by Geithman and Stinson (see [3]). They commented on the exponents in the numerator and denominator of (1), i.e. they generalized these exponents in a way that the generalized Sato production function stays linearly homogeneous. Furthermore, they commented on diminishing returns of the generalized Sato production function, but they didn't provide the proofs that the generalized Sato production function is continuous, strictly increasing and strictly quasiconcave. Thus, the first goal of this paper is to fill this gap. On the other hand, to the best of our knowledge, the solution of the cost minimization problem with the generalized Sato production function is missing in the literature, and therefore the second goal of this paper is to discuss and give the rigorous mathematical analysis of that problem.

The structure of the paper is as follows. After the introduction and the notation, the definition of the generalized Sato production function is given in the third section. In the fourth section, the preliminary facts which are necessary for further analysis are given. The first goal of the paper is disseminated in the fifth section, i.e. we show that the generalized Sato production function satisfies all properties give in Assumption 1. In the sixth section, the uniqueness of the solution of the cost minimization problem with generalized Sato production function is proved. Before the conclusions, some numerical examples are given in the seventh section.

\section{Notation}

We use the following notation from [3]: 


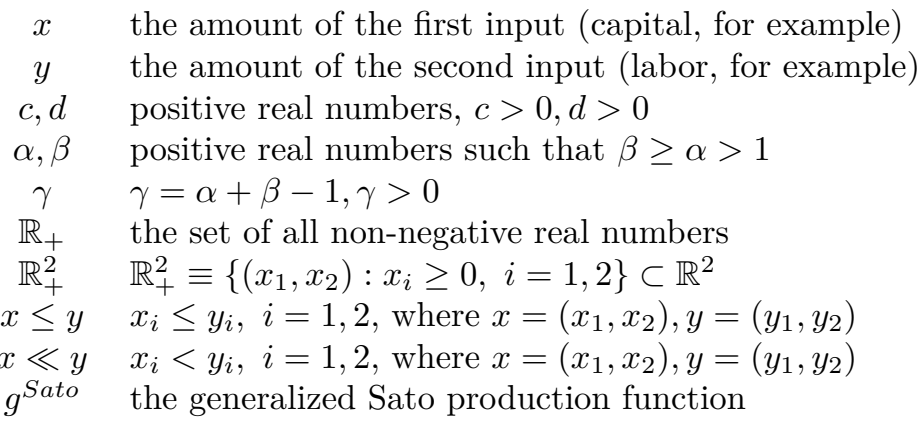

\section{The generalized Sato production function}

The generalized Sato production function, $g^{\text {Sato }}: E \cup\{(0,0)\} \subset \mathbb{R}_{+}^{2} \rightarrow \mathbb{R}_{+}$, is defined as follows:

$$
g^{\text {Sato }}(x, y)=\left\{\begin{array}{cc}
\frac{x^{\alpha} y^{\beta}}{c x^{\alpha+\beta-1}+d y^{\alpha+\beta-1}} & , \text { for }(x, y) \in E \\
0 & , \text { for } x=y=0
\end{array}\right.
$$

where

$$
\begin{array}{r}
E=\left\{(x, y) \in \mathbb{R}_{+}^{2}: \alpha d y^{\alpha+\beta-1}-c(\beta-1) x^{\alpha+\beta-1} \geq 0\right. \\
\left.\beta c x^{\alpha+\beta-1}-d(\alpha-1) y^{\alpha+\beta-1} \geq 0, x^{2}+y^{2}>0\right\}
\end{array}
$$

and

$$
c, d>0 \text { and } \beta \geq \alpha>1 \text {. }
$$

Instead of capital $K$ and labor $L$, here we use the notation $x$ and $y$, respectively. The set $E$ from (3) is called the economic region of production (see, for example, [1], [7] or [11]). All first partial derivatives of the production function are nonnegative over the economic region of production. For example, the economic region for the famous Cobb-Douglas production function is the whole first (positive) quadrant. However, it can be proved that the partial derivatives $g_{x}^{\text {Sato }}$ and $g_{y}^{\text {Sato }}$ are nonnegative over the set $E$. Since the first partial derivatives of the function (2) over the set $E$ are given by

$$
\begin{aligned}
g_{x}^{\text {Sato }}(x, y) & =\underbrace{\frac{x^{\alpha-1} y^{\beta}}{\left(c x^{\alpha+\beta-1}+d y^{\alpha+\beta-1}\right)^{2}}}_{>0} \cdot\left(\alpha d y^{\alpha+\beta-1}-c(\beta-1) x^{\alpha+\beta-1}\right), \\
g_{x}^{\text {Sato }}(x, y) & =\underbrace{\frac{x^{\alpha-1} y^{\beta}}{\left(c x^{\alpha+\beta-1}+d y^{\alpha+\beta-1}\right)^{2}}}_{>0} \cdot\left(\beta c x^{\alpha+\beta-1}-d(\alpha-1) y^{\alpha+\beta-1}\right),
\end{aligned}
$$

the set $E$ can be equivalently written as (3). Furthermore, because of (4), the following inequality holds:

$$
\frac{\beta-1}{\alpha}<\frac{\beta}{\alpha-1}
$$


By combining (3) and (7), it follows that the set $E$ can be equivalently described as

$$
E=\left\{(x, y) \in \mathbb{R}_{+}^{2} \backslash\{(0,0)\}:\left(\frac{c(\beta-1)}{\alpha d}\right)^{\frac{1}{\alpha+\beta-1}} \cdot x \leq y \leq\left(\frac{\beta c}{d(\alpha-1)}\right)^{\frac{1}{\alpha+\beta-1}} \cdot x\right\}
$$

From (8) it easily follows that $E$ is a convex set and that $E \cup\{(0,0)\}$ is a convex cone. The Figure 2 illustrates the economic region $E$ given by (8), and the Figure 2 illustrates the graph and the corresponding economic region of the function $g^{\text {Sato }}$ from (2). Let us now find the second partial derivatives of the function $g^{\text {Sato }}$. Let $\gamma=\alpha+\beta-1, \gamma>0$, and

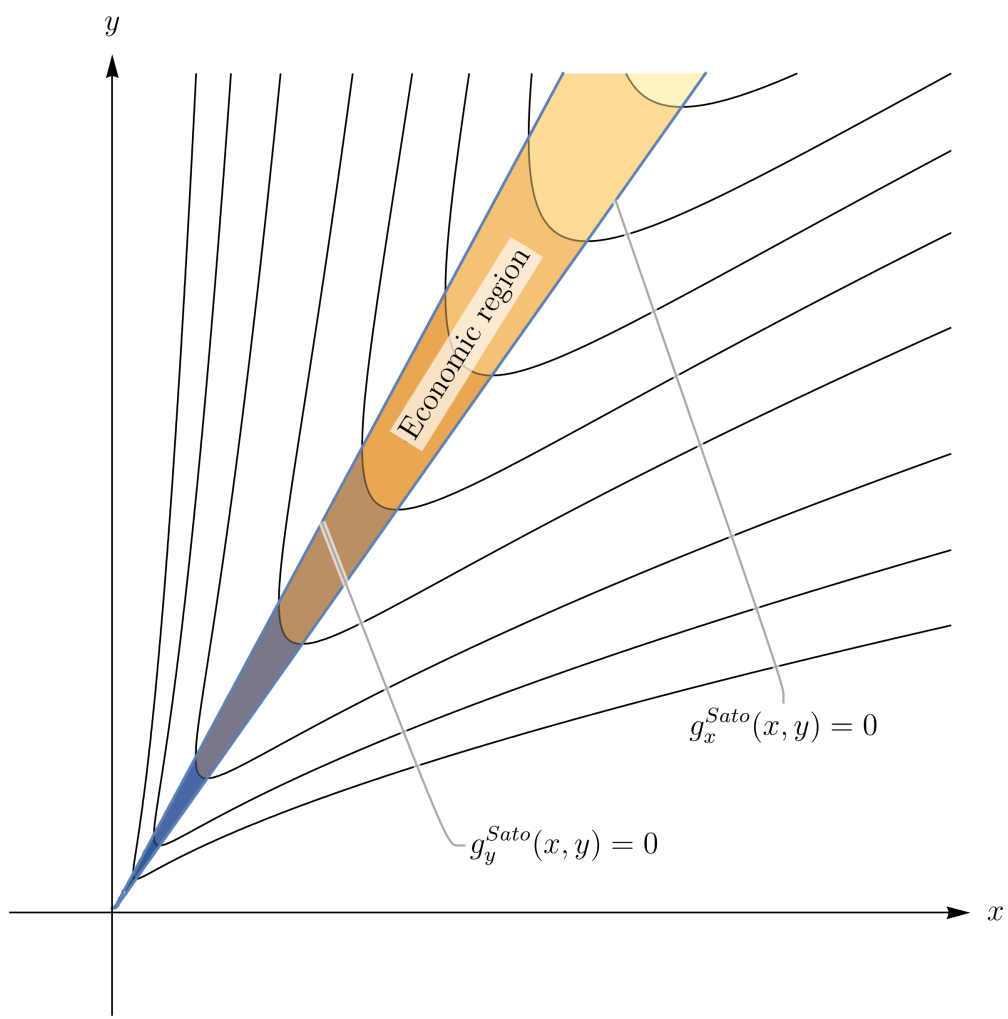

Figure 2: The illustration of the economic region $E$

$$
M(x, y)=\alpha(\alpha-1) d^{2} y^{2 \gamma}+\left(\alpha(\alpha-1)+\beta(\beta-1)-\gamma^{2}\right) c d x^{\gamma} y^{\gamma}+\beta(\beta-1) c^{2} x^{2 \gamma} .
$$

After some calculations, we have

$$
g_{x x}^{\text {Sato }}=\frac{x^{\alpha-2} y^{\beta} M(x, y)}{\left(c x^{\gamma}+d y^{\gamma}\right)^{3}}, g_{x y}^{\text {Sato }}=-\frac{x^{\alpha-1} y^{\beta-1} M(x, y)}{\left(c x^{\gamma}+d y^{\gamma}\right)^{3}}, g_{y y}^{\text {Sato }}=\frac{x^{\alpha} y^{\beta-2} M(x, y)}{\left(c x^{\gamma}+d y^{\gamma}\right)^{3}} .
$$

\section{Preliminary facts}

In order to show that the generalized Sato production function satisfies the properties from Assumption 1, we will use the weighted inequality between arithmetic mean and geometric mean (WAG-inequality) and the mean value theorem (MVT) for functions of two variables. 
The Shepard's theorem about the concavity of the homogeneous production functions will be used later, in the sixth section.

Theorem 1 (WAG-inequality). For all real numbers $\lambda_{1}, \lambda_{2}>0$ and $a_{1}, a_{2}>0$, the following inequality holds:

$$
\frac{\lambda_{1} a_{1}+\lambda_{2} a_{2}}{\lambda_{1}+\lambda_{2}} \geq a_{1}^{\frac{\lambda_{1}}{\lambda_{1}+\lambda_{2}}} a_{2}^{\frac{\lambda_{2}}{\lambda_{1}+\lambda_{2}}} \Leftrightarrow \frac{1}{\lambda_{1} a_{1}+\lambda_{2} a_{2}} \leq \frac{1}{\left(\lambda_{1}+\lambda_{2}\right) a_{1}^{\frac{\lambda_{1}}{\lambda_{1}+\lambda_{2}}} a_{2}^{\frac{\lambda_{2}}{\lambda_{1}+\lambda 2}}} .
$$

The proof of the Theorem 1 can be seen, for example, in [2].

Theorem 2 (MVT). Let $U \subseteq \mathbb{R}^{2}$ be open. Let $f: U \subseteq \mathbb{R}^{2} \rightarrow \mathbb{R}$ be a function with continuous partial derivatives $f_{x}$ and $f_{y}$. For all distinct pairs $(x, y)$ and $(u, v)$, such that the segment $[(x, y),(u, v)]$ is contained in $U$, there exists an intermediate point $(\eta, \zeta)$ on the line segment joining the points $(x, y)$ and $(u, v)$ such that

$$
f(u, v)-f(x, y)=(u-x) f_{x}(\eta, \zeta)+(v-y) f_{y}(\eta, \zeta) .
$$

The proof of the Theorem 2 can be seen, for example, in [9].

Theorem 3 (Shephard - Homogeneous production functions are concave). Let $f$ be a production function and suppose it is homogeneous of degree $\alpha \in\langle 0,1]$. Then $f$ is a concave function.

The proof of the Theorem 3 can be seen in [4].

\section{Properties of the generalized Sato production function}

In this section we show that the generalized Sato production function satisfies the properties from Assumption 1: continuity, strict monotonicity, strict quasiconcavity and the value of the function at the point zero must be the zero (see [4]). Since by definition (2) the value of the generalized Sato production function at the point zero is $g^{\operatorname{Sato}}(0,0)=0$, in this section we prove that $g^{S a t o}$ is continuous, strictly increasing and strictly quasiconcave.

Proposition 1. The generalized Sato production function is continuous.

Proof. The function $g^{\text {Sato }}$ is obviously continuous over the set $E$ (see (2)). Thus, we only have to prove that $g^{\text {Sato }}$ is continuous at the point $(0,0)$. Hence, we have to prove that for all $\epsilon>0$ there exists $\delta=\delta(\epsilon)>0$ such that for all $(x, y) \in E$ holds

$$
\sqrt{(x-0)^{2}+(y-0)^{2}}<\delta \Rightarrow\left|\frac{x^{\alpha} y^{\beta}}{c x^{\gamma}+d y^{\gamma}}-0\right|<\epsilon .
$$

For $\lambda_{1}=\alpha, \lambda_{2}=\beta, a_{1}=\frac{c x^{\gamma}}{\alpha}, a_{2}=\frac{d y^{\gamma}}{\beta}$, by using WAG-inequality (inequality (11)), we have

$$
\frac{x^{\alpha} y^{\beta}}{\alpha \cdot \frac{c x^{\gamma}}{\alpha}+\beta \cdot \frac{d y^{\gamma}}{\beta}} \leq \frac{x^{\alpha} y^{\beta}}{(\alpha+\beta)\left(\frac{c x^{\gamma}}{\alpha}\right)^{\frac{\alpha}{\alpha+\beta}}\left(\frac{d y^{\gamma}}{\beta}\right)^{\frac{\beta}{\alpha+\beta}}}=\frac{\left(x^{\alpha} y^{\beta}\right)^{\frac{1}{\alpha+\beta}}}{(\alpha+\beta)\left(\frac{c x^{\gamma}}{\alpha}\right)^{\frac{\alpha}{\alpha+\beta}}\left(\frac{d y^{\gamma}}{\beta}\right)^{\frac{\beta}{\alpha+\beta}}}
$$


For any arbitrary but fixed $\epsilon>0$, let

$$
\delta=\delta(\epsilon)=(\alpha+\beta)\left(\frac{c}{\alpha}\right)^{\frac{\alpha}{\alpha+\beta}}\left(\frac{d}{\beta}\right)^{\frac{\beta}{\alpha+\beta}} \cdot \epsilon .
$$

Suppose that $\sqrt{x^{2}+y^{2}}<\delta$. Since $x<\sqrt{x^{2}+y^{2}}<\delta$ and $y<\sqrt{x^{2}+y^{2}}<\delta$, we have

$$
\begin{aligned}
\left|g^{\text {Sato }}(x, y)-0\right| & =\frac{x^{\alpha} y^{\beta}}{c x^{\gamma}+d y^{\gamma}} \leq \frac{\left(x^{\alpha} y^{\beta}\right)^{\frac{1}{\alpha+\beta}}}{(\alpha+\beta)\left(\frac{c}{d}\right)^{\frac{\alpha}{\alpha+\beta}}\left(\frac{d}{\beta}\right)^{\frac{\beta}{\alpha+\beta}}} \\
& \leq \frac{\delta^{\frac{\alpha}{\alpha+\beta}} \cdot \delta^{\frac{\beta}{\alpha+\beta}}}{(\alpha+\beta)\left(\frac{c}{d}\right)^{\frac{\alpha}{\alpha+\beta}}\left(\frac{d}{\beta}\right)^{\frac{\beta}{\alpha+\beta}}}=\frac{(\alpha+\beta)\left(\frac{c}{d}\right)^{\frac{\alpha}{\alpha+\beta}}\left(\frac{d}{\beta}\right)^{\frac{\beta}{\alpha+\beta}}}{(\alpha+\beta)\left(\frac{c}{d}\right)^{\frac{\alpha}{\alpha+\beta}}\left(\frac{d}{\beta}\right)^{\frac{\beta}{\alpha+\beta}}}=\epsilon,
\end{aligned}
$$

which proves (13). This completes the proof.

Proposition 2. The generalized Sato production function is strictly increasing.

Proof. We have to prove that for any two points $\left(x_{1}, y_{1}\right),\left(x_{2}, y_{2}\right) \in E \cup\{(0,0)\}$ the following implication holds:

$$
\left(x_{1}, y_{1}\right) \ll\left(x_{2}, y_{2}\right) \Rightarrow g^{\text {Sato }}\left(x_{1}, y_{1}\right)<g^{\text {Sato }}\left(x_{2}, y_{2}\right)
$$

Since $g_{x}^{\text {Sato }}(x, y)>0, g_{y}^{\text {Sato }}>0$ for all $(x, y) \in \operatorname{int} E$, by Theorem 2, there exists the point $(\eta, \zeta)$ on the line segment joining the points $\left(x_{1}, y_{1}\right)$ and $\left(x_{2}, y_{2}\right)$ such that

$$
g^{\text {Sato }}\left(x_{2}, y_{2}\right)-g^{\text {Sato }}\left(x_{1}, y_{1}\right)=\underbrace{\left(x_{2}-x_{1}\right)}_{>0} \underbrace{g_{x}^{\text {Sato }}(\eta, \zeta)}_{>0}+\underbrace{\left(y_{2}-y_{1}\right)}_{>0} \underbrace{g_{y}^{\text {Sato }}(\eta, \zeta)}_{>0} .
$$

Obviously, from (18) follows (17). This completes the proof.

Proposition 3. The generalized Sato production function is strictly quasiconcave.

Proof. Since $E \cup\{(0,0)\}$ is a convex cone, it is sufficient to prove that the determinant of the bordered Hessian of the function $g^{\text {Sato }}$ in any point $(x, y) \in \operatorname{int} E$ is positive, i.e.

$$
\begin{aligned}
\operatorname{det} H_{g^{\text {Sato }}}(x, y) & =\left|\begin{array}{ccc}
0 & g_{x}^{\text {Sato }} & g_{y}^{\text {Sato }} \\
g_{x}^{\text {Sato }} & g_{x x}^{\text {Sato }} & g_{y x}^{\text {Sato }} \\
g_{y}^{\text {Sato }} & g_{x y}^{\text {Sato }} & g_{y y}^{\text {Sato }}
\end{array}\right|= \\
& =2 g_{x}^{\text {Sato }} g_{y}^{\text {Sato }} g_{x y}^{\text {Sato }}-\left(g_{x}^{\text {Sato }}\right)^{2} g_{y y}^{\text {Sato }}-\left(g_{y}^{\text {Sato }}\right)^{2} g_{x x}^{\text {Sato }}>0
\end{aligned}
$$

After some calculation, we find

$$
\operatorname{det} H_{g}^{\text {Sato }}(x, y)=-\underbrace{\frac{x^{3 \alpha-2} y^{3 \beta-2}}{\left(c x^{\alpha+\beta-1}+d y^{\alpha+\beta-1}\right)^{5}}}_{>0} \cdot M(x, y),
$$


where $M(x, y)$ is given by $(9)$. Let

$$
t=\frac{d}{c} \cdot \frac{y^{\gamma}}{x^{\gamma}}
$$

Then $M(x, y)$ can be written as

$$
M(x, y)=c^{2} x^{2 \gamma} h(t)
$$

where

$$
h(t)=\alpha(\alpha-1) t^{2}+\left(\alpha(\alpha-1)+\beta(\beta-1)-\gamma^{2}\right) t+\beta(\beta-1) .
$$

From (8), it follows that $\frac{\beta-1}{\alpha}<t<\frac{\alpha}{\beta-1}$, and, since $\alpha(\alpha-1)>0$, we have $h(t)<0$ (see Figure $3)$. Thus, from (20)-(23), we get that for any point $(x, y) \in \operatorname{int} E$ determinant of the bordered Hessian is positive, i.e.

$$
\operatorname{det} H_{g^{\text {Sato }}}(x, y)=-\underbrace{\frac{x^{3 \alpha-2} y^{3 \beta-2}}{\left(c x^{\alpha+\beta-1}+d y^{\alpha+\beta-1}\right)^{5}}}_{>0} \cdot \underbrace{c^{2} x^{2 \gamma}}_{>0} \cdot \underbrace{h(t)}_{<0}>0 .
$$

This completes the proof.

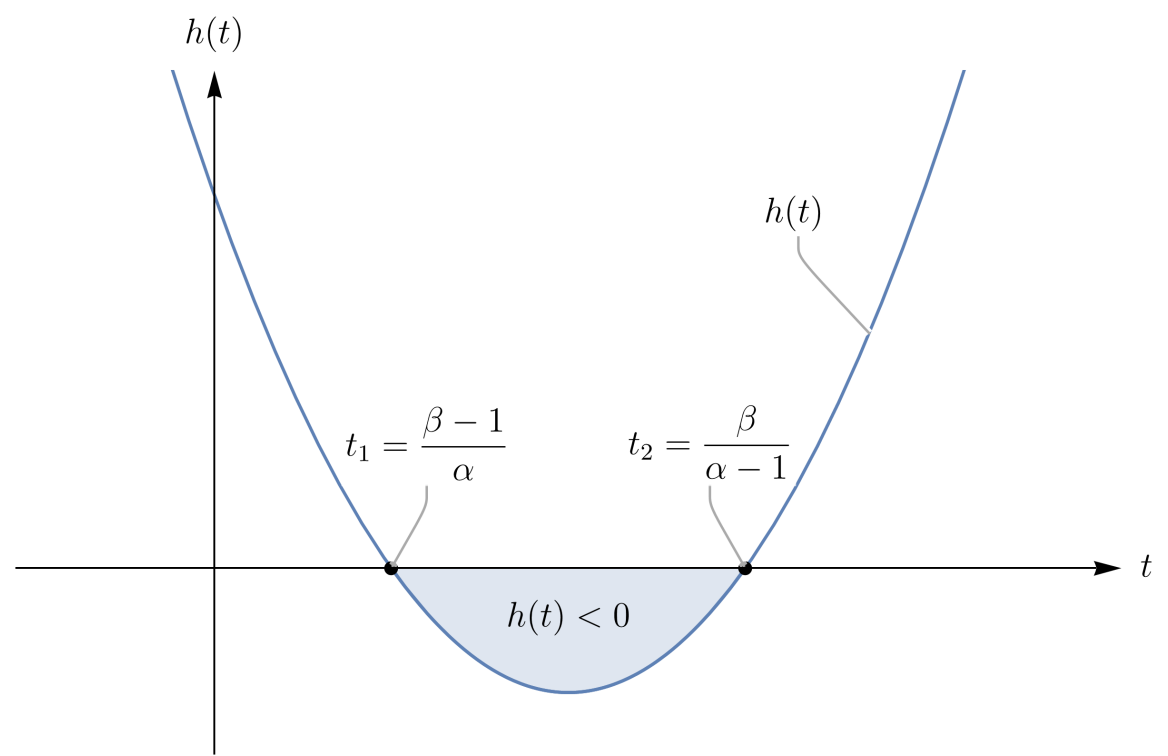

Figure 3: The graph of the function $h(t)$ from (23) is parabola

Finally, after proving Proposition 1, Proposition 2 and Proposition 3, we can conclude that the generalized Sato production function from (2) satisfies all properties from Assumption 1.

\section{The cost minimization problem with the generalized Sato production function}

In this section, we analyse the cost minimization problem with the generalized Sato production function. Let $w_{1}>0$ and $w_{2}>0$ be the prices of the first and the second input, respectively, 
while $q>0$ is the amount of the production. Then, the problem can be formulated as

$$
\begin{array}{lrl}
\min _{x, y \geq 0} & f(x, y)=w_{1} x+w_{2} y \\
\text { s.t. } & g^{\text {Sato }}(x, y)=\frac{x^{\alpha} y^{\beta}}{c x^{\alpha+\beta-1}+d y^{\alpha+\beta-1}} \geq q .
\end{array}
$$

Since $g^{\text {Sato }}$ is strictly increasing, the constraint will be binding at a solution. Thus, the problem (25) can be equivalently formulated as

$$
\begin{array}{lr}
\min _{x, y \geq 0} & f(x, y)=w_{1} x+w_{2} y \\
\text { s.t. } & g^{\text {Sato }}(x, y)=\frac{x^{\alpha} y^{\beta}}{c x^{\alpha+\beta-1}+d y^{\alpha+\beta-1}}=q .
\end{array}
$$

We solve the problem (26) by using the standard Lagrange multiplier method for constrained optimization. The corresponding Lagrangian function is

$$
L(x, y, \lambda)=w_{1} x+w_{2} y+\lambda\left(g^{\text {Sato }}(x, y)-q\right) .
$$

From the first order conditions, we have

$$
\left\{\begin{array}{l}
0=L_{x}=w_{1}+\lambda g_{x}^{\text {Sato }} \Rightarrow w_{1}=-\lambda g_{x}^{\text {Sato }} \\
0=L_{y}=w_{2}+\lambda g_{y}^{\text {Sato }} \Rightarrow w_{2}=-\lambda g_{y}^{\text {Sato }} \\
q=\frac{x^{\alpha} y^{\beta}}{c x^{\alpha+\beta-1}+d y^{\alpha+\beta-1}}
\end{array}\right.
$$

Since the function $g^{\text {Sato }}$ from (2) is a linearly homogeneous and strictly quasiconcave function, by Theorem 3 we conclude that $g^{\text {Sato }}$ is strictly concave. That means that it is enough to find the solution of the system (28), since the first order conditions are sufficient in this case. From (28), we have

$$
\frac{w_{1}}{w_{2}}=\frac{g_{x}^{\text {Sato }}}{g_{y}^{\text {Sato }}}=\frac{y}{x} \cdot \frac{c(1-\beta) x^{\alpha+\beta-1}+\alpha d y^{\alpha+\beta-1}}{\beta c x^{\alpha+\beta-1}+d(1-\alpha) y^{\alpha+\beta-1}} .
$$

Let $w=\frac{w_{1}}{w_{2}}, t=\frac{d}{c} \cdot \frac{y^{\alpha+\beta-1}}{x^{\alpha+\beta-1}}, u(t)=\frac{w^{1-\alpha-\beta} c}{d} t$ and $v(t)=\left(\frac{(1-\alpha) t+\beta}{\alpha t+(1-\beta)}\right)^{\alpha+\beta-1}$. Then, from $(29)$ we have

$$
u(t)=v(t) .
$$

The domain of the function $v$ is $D_{v}=\left\langle\frac{\beta-1}{\alpha}, \frac{\beta}{\alpha-1}\right\rangle$. Since the function $v$ is strictly decreasing (because its first derivative is negative), i.e.

$$
v^{\prime}(t)=\underbrace{(\alpha+\beta-1)}_{>0}\left(\frac{(1-\alpha) t+\beta}{\alpha t+(1-\beta)}\right)^{\alpha+\beta-2} \cdot \frac{\overbrace{1-\alpha-\beta}^{<0}}{(\alpha t+(1-\beta))^{2}}<0,
$$

and the function $u$ is strictly increasing, the graphs of the functions $u$ and $v$ intersect only once (see Figure 4). From here, we conclude that the equation (30) has a unique solution, which further implies that the problem (26) has unique solution over the set $E$ (see Figure 5). 


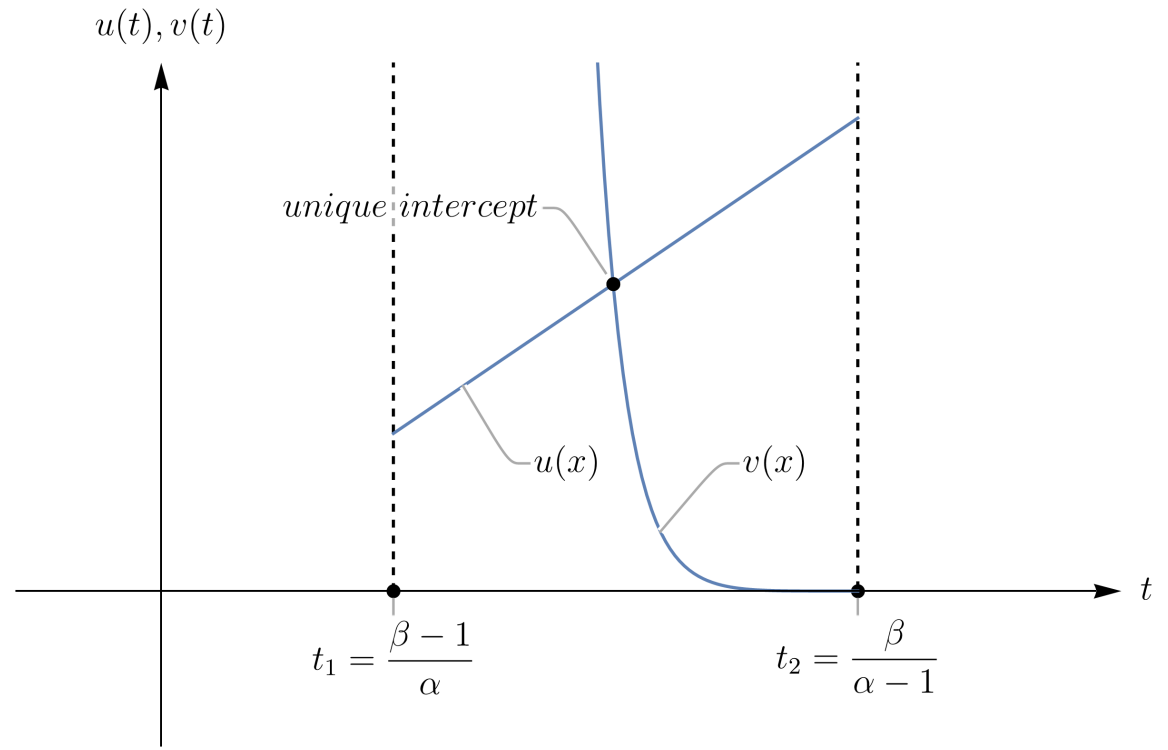

Figure 4: The intersection of the graphs of the functions $u$ and $v$

The uniqueness of the solution of the problem (26) implies that the corresponding cost function $c\left(w_{1}, w_{2}, q\right)$ is well defined. However, the explicit closed-form solution of the problem (26) in terms of general parameters $w_{1}, w_{2}$, and $q$ is not foundable.
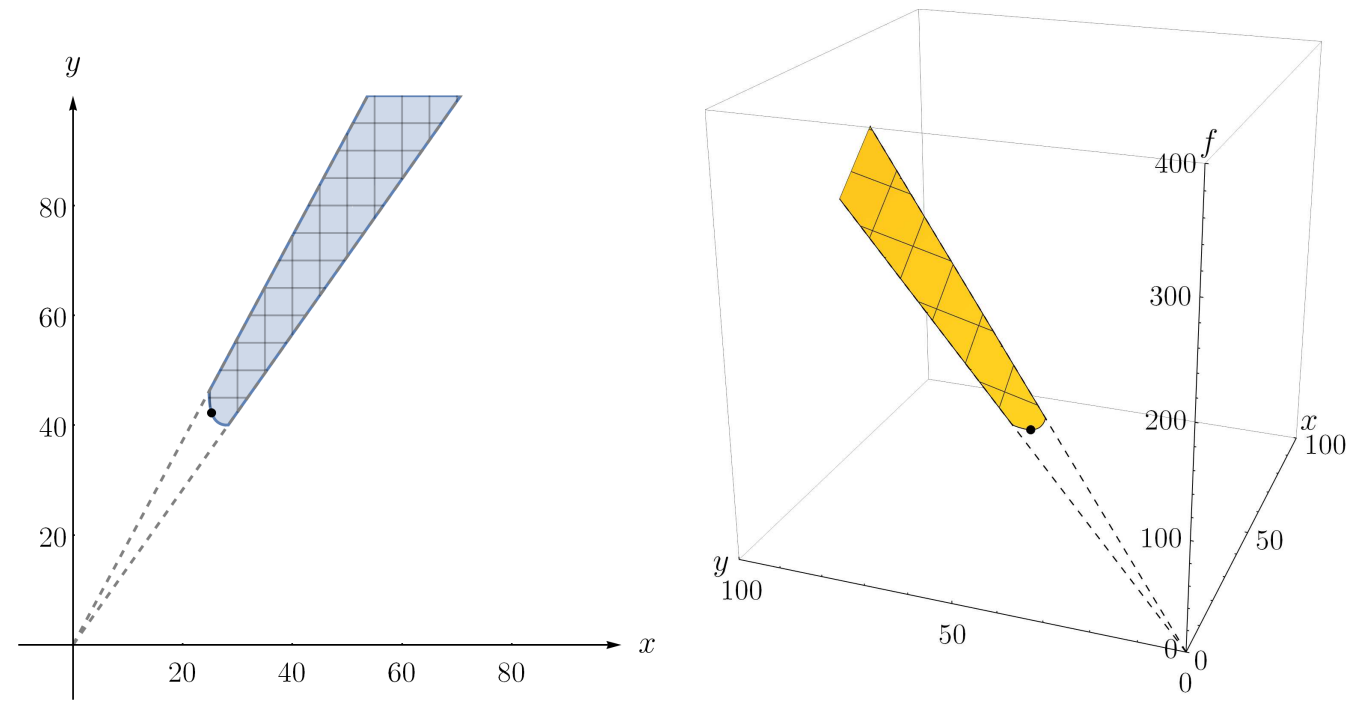

Figure 5: The unique solution of the problem (26) over the set $E$

\section{Numerical examples}

Since the explicit solution of the problem (26) in terms of $w_{1}, w_{2}$, and $q$ cannot be found, there is no explicit formula for the corresponding cost function and conditional input demands which are functions of $w_{1}, w_{2}$, and $q$. However, let us examine two numerical examples.

Example 1. Let $\alpha=2, \beta=3, c=4, d=1, w_{1}=3, w_{2}=1, q=10$. Then the problem (26) 
becomes

$$
\begin{array}{rl}
\min _{x, y \geq 0} & f(x, y)=3 x+y \\
\text { s.t. } & g^{\text {Sato }}(x, y)=\frac{x^{2} y^{3}}{4 x^{4}+y^{4}}=10 .
\end{array}
$$

The optimal solution of the problem (32) is $x^{*}=25.2863, y^{*}=42.2377, f_{\min }^{*}=118.097$ (see Figures 6 and 7 ).

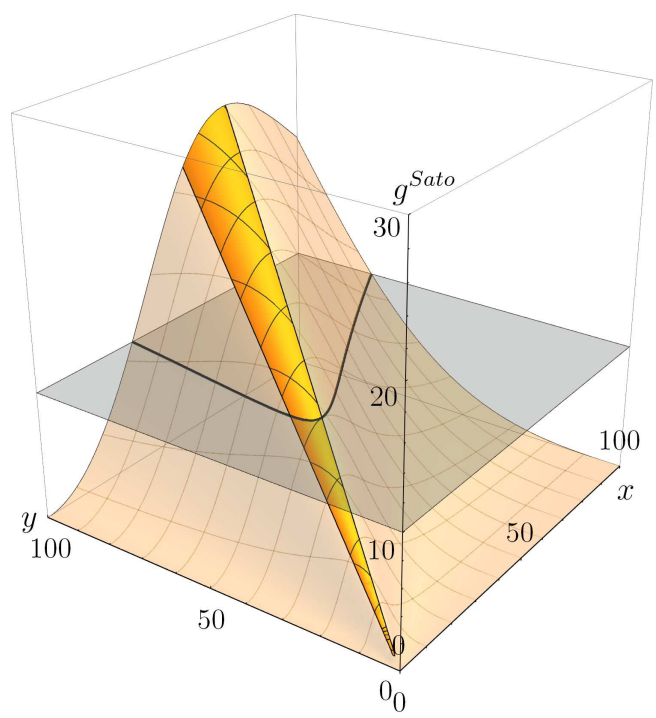

Figure 6: The function $g^{\text {Sato }}(x, y)=\frac{x^{2} y^{3}}{4 x^{4}+y^{4}}$ with economic region and isoquant $q=10$
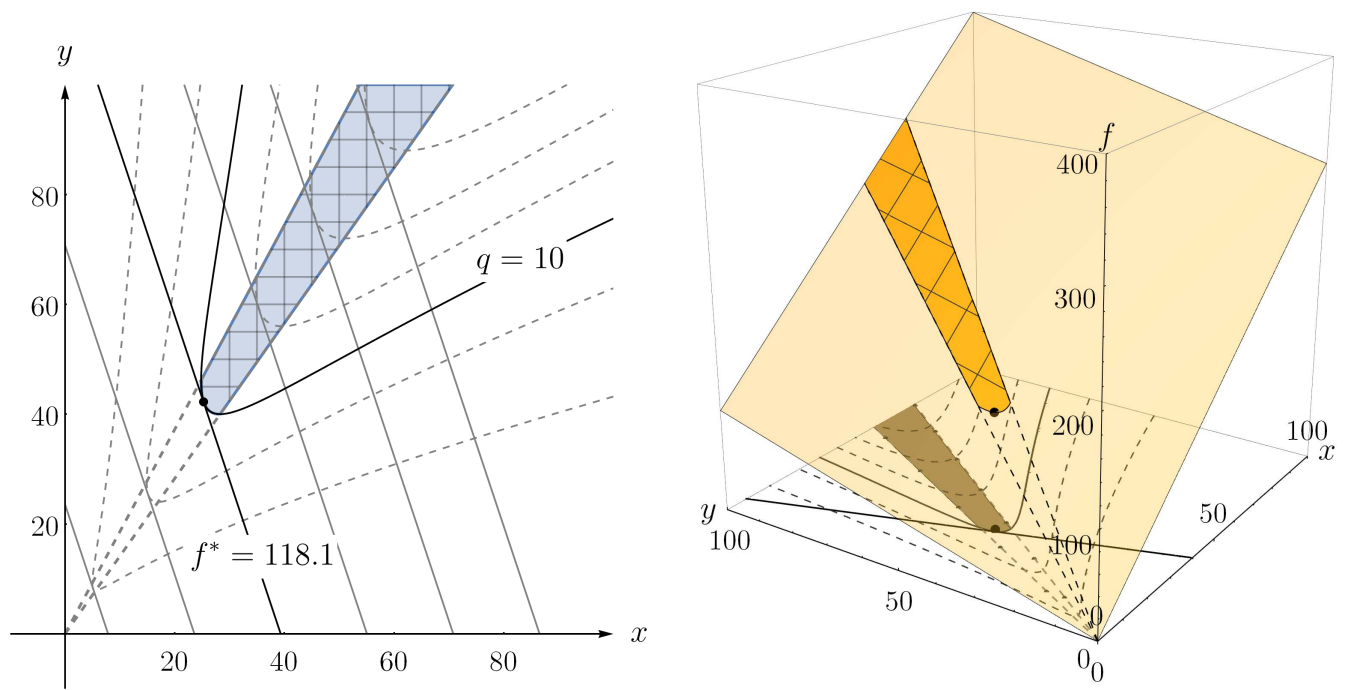

Figure 7: The function $g^{\text {Sato }}(x, y)=\frac{x^{2} y^{3}}{4 x^{4}+y^{4}}$ with economic region and $\frac{x^{2} y^{3}}{4 x^{4}+y^{4}} \geq 10$. Black point is the optimal one 
Example 2. Let $\alpha=\beta, c=d, w_{1}=w_{2}, q>0$. Then the problem (26) becomes

$$
\begin{array}{rl}
\min _{x, y \geq 0} & f(x, y)=w_{1} x+w_{1} y \\
\text { s.t. } & g^{\text {Sato }}(x, y)=\frac{x^{\alpha} y^{\alpha}}{c x^{\alpha}+c y^{\alpha}}=q .
\end{array}
$$

Equation (30) becomes

$$
t=\left(\frac{(1-\alpha) t+1}{\alpha t+(1-\alpha)}\right)^{2 \alpha-1},
$$

which has a unique solution $t^{*}=1$. From here, we have $y^{*}=x^{*}$. By substituting $y=x$ into (33), we get $y^{*}=x^{*}=2 c q$. Now, it is easy to find the minimum of the problem (33), i.e. it is easy to find the cost function and the corresponding conditional input demands:

$$
\begin{aligned}
& c\left(w_{1}, q\right)=w_{1} \cdot 2 c q+w_{1} \cdot 2 c q=4 c w_{1} q \\
& x\left(w_{1}, q\right)=2 c q \\
& y\left(w_{1}, q\right)=2 c q .
\end{aligned}
$$

\section{Conclusion}

In microeconomics, besides the famous Cobb-Douglas and CES production function, the generalized Sato production function was proposed in 1960's as a new form of production function. However, to the best of our knowledge, the proofs that the generalized Sato production function satisfies four properties - continuity, strict monotonicity, strict quasiconcavity and the value of the function at the point zero must be the zero, are missing in literature. So, as fulfilling the first goal of this paper, we have given the rigorous proofs that the generalized Sato production function is continuous, strictly increasing and strictly quasiconcave over its domain. Moreover, we have treated the cost minimization problem with generalized Sato production function and proved that the problem has a unique solution, which was the second goal of this paper. This result implies the existence of the corresponding cost function and conditional input demands, which is an important result in microeconomic theory. For the future research, the sensitivity analysis and some comparative analysis of the chosen generalized Sato production function as opposed to others more often used in the applied work can be given. Also, geometric programming can be applied to solve the cost minimization problem with generalized Sato production function in a more elegant way.

\section{References}

[1] Besanko, D. and Braeutigam, R. R. (2014). Microeconomics. Fifth Edition. Wiley. https://www.wiley.com/en-us/Microeconomics\%2C+5th+Edition-p-9781118883228

[2] Cvetkovski, Z. (2012). Inequalities. Berlin: Springer. doi: 10.1007/978-3-642-23792-8

[3] Geithman, D. T. and Stinson, B. S. (1969). A note on Diminishing Returns and linear homogeneity. The American Economist, 13 (1), 77-79. doi: 10.1177/056943456901300111

[4] Jehle, G. A. and Reny, P. J. (2011). Advanced Microeconomic Theory. Third Edition. Pearson.

[5] Kojić, V., Lukač, Z. and Puljić, K. (2019). On the properties of the Sato production function. Proceedings of the $15^{\text {th }}$ International Symposium on Operational Research, Bled, Slovenia, 599-604. http://fgg-web.fgg.uni-lj.si/ /sdrobne/sor/SOR'19\%20-\%20Proceedings.pdf

[6] Mas-Colell, A. and Whinston, M. D., Green, J. R. (1995). Microeconomic Theory. New York: Oxford University Press.

[7] Mundlak, Y. (1958). A Note on the Symmetry of Homogeneous Production Function and the Three Stages of Production. Journal of Farm Economics, 40 (3), 756-761. doi: 10.2307/1235389 
[8] Roubalová, L., Hampel, D. and Viskotová, L. (2018). Technological Progress at the Sectoral Level: the Sato Production Function Approach. Proceedings of the $36^{\text {th }}$ International Conference Mathematical Methods in Economics, Jindřichův Hradec, Czech Republic, 470-475. https://mme2018.fm.vse.cz/wp-content/uploads/2018/09/MME2018-Electronic_proceedings.pdf

[9] Sahoo, P. K. and Riedel, T. (1998). Mean Value Theorems and Functional Equations. Singapore: World Scientific Publishing.

[10] Sato, R. (1964). Diminishing Returns and Linear Homogeneity: Comment. The American Economic Review, 54 (5), 744-745. https://www.jstor.org/stable/1818574

[11] Seagraves, J. A. and Pasour, E. C. (Jr.) (1969). On Defining Uneconomic Regions of the Production Function. American Journal of Agricultural Economics, 51 (1), 195-202. doi: 10.2307/1238321 